\title{
Being Quantitative in Reciprocal Space
}

\author{
L. D. Marks
}

Department of Materials Science and Engineering, Northwestern University, Evanston, IL 60201

If we knew the positions of all the electrons there would be no need to find the atoms

Over the years x-ray crystallography has become adept in handling numbers from diffraction and converting these into quantitative structural results. In most cases they have no alternative as images are not available. Different from this electron microscopists have been adept at doing crystallography in real space using images, but often this is where the analysis stops. However, we do have numbers from images which can be used (at least in theory [1-3]) to measure quantitative parameters such as local charge density changes, although this is easier to do with diffraction data either using precession [4] or just from diffraction such as at surfaces [5-9]

The focus of this paper will be on the issue of how quantitative can one be, and what can this give you particularly in reciprocal space. Examples will range from fairly simple concepts such as using precession electron diffraction to determine charge density (Figure 1) to more complicated examples such as refining surface charge density (Figure 2) and being able to combine real and reciprocal space methods such as HREM or STM with diffraction to solve complicated structures such as the homologous series of reconstructions on the (110) surface of $\mathrm{SrTiO}_{3}$ [8] (Figure 3) or large unit cell reconstructions such as the $\sqrt{ } 13 x \sqrt{ } 13$ reconstruction on $\mathrm{SrTiO}_{3}(001)$ [9] (Figure 4).

\section{References}

[1] Ciston, J., J.S. Kim, S.J. Haigh, A.I. Kirkland, and L.D. Marks, Optimized conditions fordirect imaging of bonding charge density in electron microscopy. Ultramicroscopy, 2011. In press: p. http://dx.doi.org/10.1016/j.ultramic.2010.12.003.

[2] Deng, B., L.D. Marks, and J.M. Rondinelli, Charge defects glowing in the dark. Ultramicroscopy, 2007. 107(4-5): p. 374-381.

[3] Deng, B. and L.D. Marks, Theoretical structure factors for selected oxides and their effects in high-resolution electron-microscope (HREM) images. Acta Crystallographica Section A,2006. 62: p. 208-216.

[4] Ciston, J., B. Deng, L.D. Marks, C.S. Own, and W. Sinkler, A quantitative analysis of thecone-angle dependence in precession electron diffraction. Ultramicroscopy, 2008. 108(6): p.514-522.

[5] Ciston, J., A. Subramanian, I.K. Robinson, and L.D. Marks, Diffraction refinement of localized antibonding at the Si(111) $7 x 7$ surface. Physical Review B, 2009. 79(19): p.193302.

[6] Marks, L.D., J. Ciston, B. Deng, and A. Subramanian, Fitting valence charge densities at a crystal surface. Acta Crystallographica Section A, 2006. 62: p. 309-315.

[7] Ciston, J., L.D. Marks, R. Feidenhans'l, O. Bunk, G. Falkenberg, and E.M. Lauridsen, Experimental surface charge density of the Si (100)-2xlH surface. Physical Review B, 2006.74(8): p. 085401.

[8] Enterkin, J.A., A.K. Subramanian, B.C. Russell, M.R. Castell, K.R. Poeppelmeier, and L.D. Marks, A homologous series of structures on the surface of $\mathrm{SrTiO}_{3}(110)$. Nature Materials, 2010. 9(3): p. 245-248.

[9] Kienzle, D.M., A.E. Becerra-Toledo, and L.D. Marks, Structure of the R13 Reconstruction on $\mathrm{SrTiO}_{3}$ (001). Submitted, 2011. 


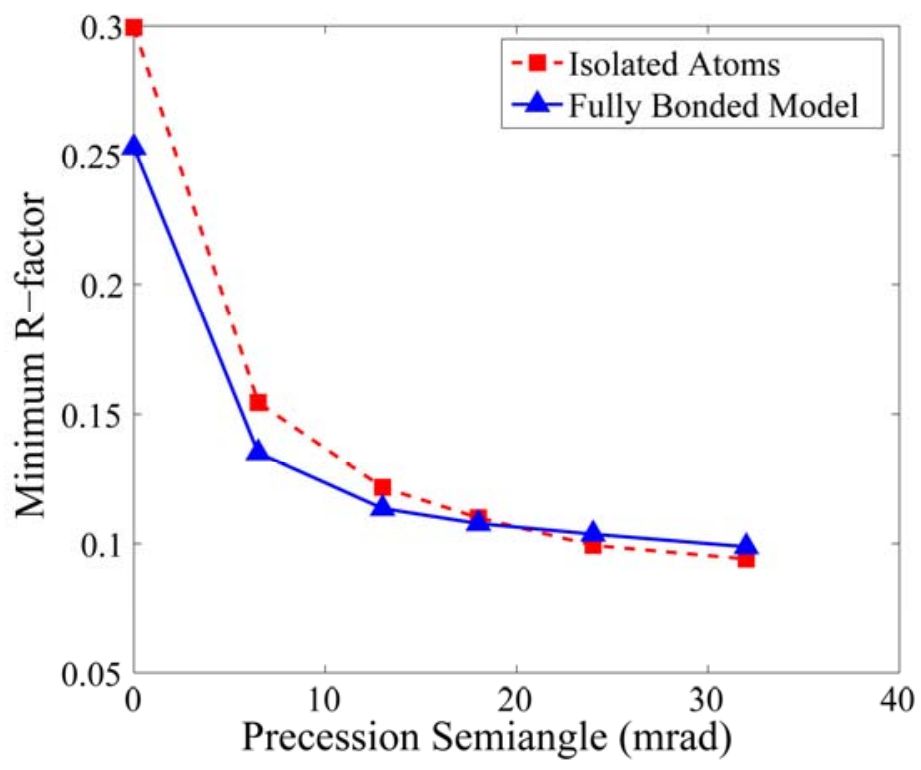

FIG. 1. Comparison of R1 fit for Andalusite precession diffraction data as a function of semiangle using structure factors calculated using the independent atom model (red) versus those calculated using the all-electron DFT code Wien $2 k$. The intensities were in both cases calculated fully dynamically using a modified multislice code.

FIG. 2. Pseudocolor plot of the charge density for the Si (001) 2x1 surface on the left calculated using DFT and on the right from a pseudo-atom refinement versus experimental data. The match is fairly good, with the experimental data being less sharp due to thermal vibrations as well as measurement errors.

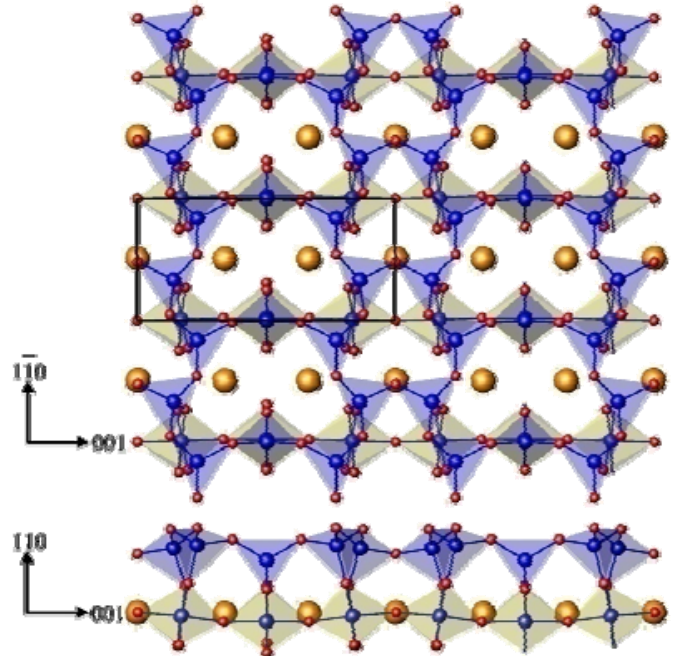

FIG. 4. Experimental structure of the $\sqrt{ } 13 x \sqrt{ } 13$ reconstruction on $\mathrm{SrTiO}_{3}$ (001) showing $\mathrm{TiO}_{5}[]$ octahedron in green as well as the full $\mathrm{TiO}_{6}$ octahedron in purple.

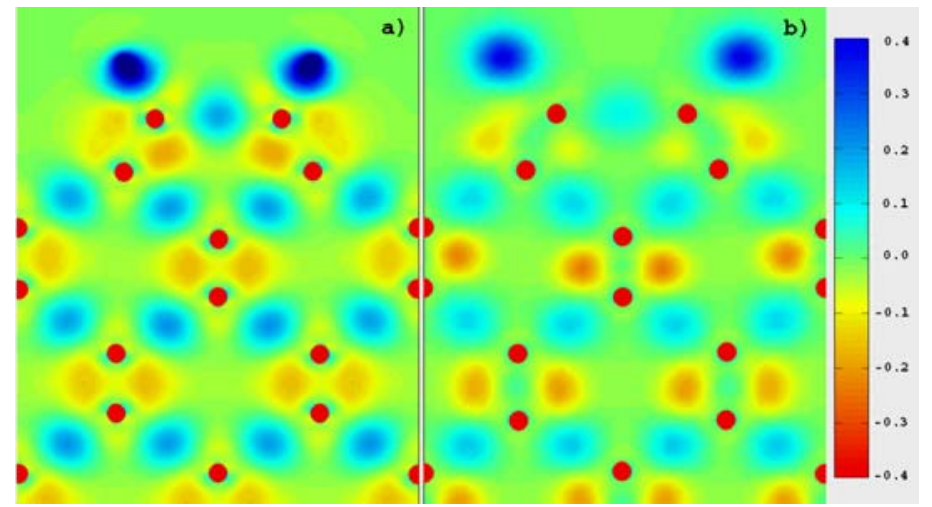

FIG. 3. Diagram of the $3 \times 1$ reconstruction of the $\mathrm{SrTiO}_{3}$ (110) surface viewed from above (top) and from the side (bottom). Shown in blue are the rings of $\mathrm{TiO}_{4}$ tetrahedra in the outermost surface plane, with the $\mathrm{TiO}_{6}$ octrahedra as grew and the $\mathrm{Sr}$ atoms yellow.

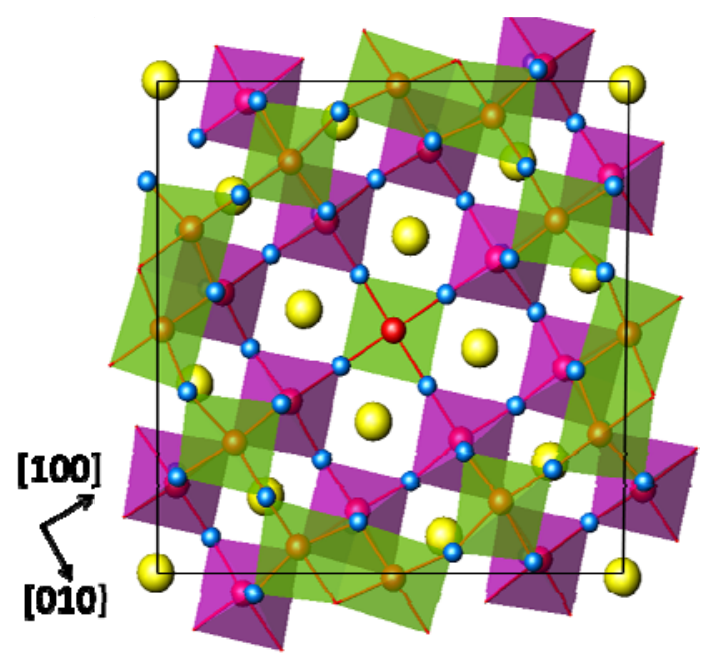

